\title{
BMJ Open Does care in a specialised stroke prevention clinic improve poststroke blood pressure control: a protocol for a randomised comparative effectiveness study
}

\author{
Anjail Z Sharrief, ${ }^{1,2}$ Evelyn Hinojosa, ${ }^{1}$ Gail Cooksey, ${ }^{1}$ Munachi N Okpala, ${ }^{1}$ \\ Elenir B Avritscher, ${ }^{3}$ Claudia Pedroza, ${ }^{3}$ Mary Carter Denny, ${ }^{4}$ Joshua Samuels, ${ }^{3}$ \\ Jon E Tyson, ${ }^{3}$ Sean I Savitz ${ }^{1}$
}

To cite: Sharrief AZ, Hinojosa E, Cooksey G, et al. Does care in a specialised stroke prevention clinic improve poststroke blood pressure control: a protocol for a randomised comparative effectiveness study. BMJ Open 2019:9:e024695. doi:10.1136/ bmjopen-2018-024695

- Prepublication history for this paper is available online. To view these files, please visit the journal online (http://dx.doi. org/10.1136/bmjopen-2018024695).

Received 18 June 2018 Revised 30 August 2018 Accepted 26 September 2018

Check for updates

(c) Author(s) (or their employer(s)) 2019. Re-use permitted under CC BY-NC. No commercial re-use. See rights and permissions. Published by BMJ.

For numbered affiliations see end of article.

Correspondence to

Dr Anjail Z Sharrief;

anjail.z.sharrief@uth.tmc.edu

\section{ABSTRACT}

Introduction Hypertension is a major risk factor for recurrent stroke, and blood pressure $(\mathrm{BP})$ reduction is associated with decreased risk of stroke recurrence. However, many stroke survivors have poorly controlled BP after their initial stroke. The Stroke Transitions Education and Prevention (STEP) Clinic was established to provide a comprehensive approach to stroke risk factor reduction. Methods and analysis This randomised comparative effectiveness study was designed to assess the impact of care in the STEP clinic versus usual care on poststroke $\mathrm{BP}$ reduction. Eligible hospitalised patients with ischaemic stroke, haemorrhagic stroke or transient ischaemic attack are scheduled for a clinic screening visit within 4 weeks of discharge if they meet baseline inclusion criteria. At the clinic visit, patients who have uncontrolled $\mathrm{BP}$, defined as automated office $B P \geq 135 / 85 \mathrm{~mm} \mathrm{Hg}$ are randomised (1:1) to either the STEP clinic or usual care for management. STEP clinic patients receive instructions to self-monitor, a BP monitor, sleep apnoea screening, dietary counselling, review of BP monitoring records and adjustment of medications. Patients are followed by a neurologist and a stroke-trained nurse practitioner. Usual care participants are seen by a neurologist and recommendations for secondary prevention are sent to primary care providers. The primary outcome is the difference in mean daytime ambulatory systolic BP at 6 months, assessed using linear regression analysis. Secondary outcomes include 24 hours ambulatory BP, medication adherence and medication selfefficacy, and composite cardiovascular events.

Ethics and dissemination This study was approved by the Institutional Review Boards at the McGovern Medical School at the University of Texas Health Sciences Center and the Georgetown University School of Medicine.

Uninsured and Spanish-speaking patients are included in the study.

Trial registration number NCT02591394; Pre-results.

\section{INTRODUCTION}

Improvements in stroke prevention, acute treatment and organised systems of care for
Strengths and limitations of this study

- This study is designed as a randomised controlled trial of an organisational intervention aimed at improving blood pressure (BP) control after stroke. This is a high-priority area for stroke prevention.

- This study will include a diverse patient population as we anticipate that more than $30 \%$ of eligible patients will be African-American who have higher risk for uncontrolled BP and stroke recurrence.

- We will use Bayesian analysis which will allow us to estimate the probability that the intervention is effective at reducing $B P$.

- We are including uninsured and underserved patients and may therefore have higher attrition rates.

- The trial was designed for patients with mild to moderate poststroke disability, and results may not be generalisable to patients with more severe strokes.

acute stroke are all thought to contribute to declines in stroke mortality observed over the past decade. ${ }^{1}$ Nevertheless, there are over 7 million stroke survivors in the USA. With increasing survival after stroke and expected increases in stroke incidence related to population ageing, the prevalence of stroke is projected to increase by 3.4 million in $2030 .^{23}$ Despite these projections, there has been little emphasis or research on organising systems of care for stroke survivors.

Poststroke care should address the unique needs of stroke survivors and prioritise risk factor management for prevention of recurrent stroke. Stroke risk increases after incident stroke, and $25 \%$ of incident strokes are recurrent events. ${ }^{2}$ Recurrent stroke carries additional risk of morbidity and mortality compared with the incident stroke. ${ }^{4}$ Quantitative modelling suggests that up to $80 \%$ 
of vascular events after stroke can be prevented by addressing modifiable risk factors through pharmacological and behavioural interventions. ${ }^{5}$

Hypertension is the most important risk factor for ischaemic stroke and haemorrhagic stroke and reduction in blood pressure (BP) after stroke is associated with markedly reduced risk of stroke recurrence.$^{6-8}$ However, available data suggest that hypertension remains poorly controlled after the incident stroke. A report from the Reasons for Geographic and Racial Differences in Stroke (REGARDS) study revealed that risk factor awareness and control were poor in participants who self-reported a history of stroke. ${ }^{9}$ Stroke survivors were more likely to have undiagnosed hypertension and poorly controlled BP compared with those without prior stroke. Only $66.7 \%$ of stroke survivors had controlled BP, and African-American stroke survivors were more likely to have undiagnosed hypertension and uncontrolled hypertension (among those treated) than white stroke survivors.

The REGARDs data are supported by other studies that confirm prior ischaemic or haemorrhagic stroke. Baseline visit data from the Secondary Prevention of Small Subcortical Strokes (SPS3) trial showed that $56 \%$ of ischaemic stroke survivors in the USA who participated in the study did not have controlled BP $2 \frac{1 / 2}{2}$ months after stroke. ${ }^{10}$ African-Americans were more likely to have poorly controlled BP than white Americans in the subset of US participants in SPS3. ${ }^{10}$ Investigators from the DiffErenCes in the Imaging of Primary Hemorrhage based on Ethnicity or Race project, an observational cohort study based in Washington DC, demonstrated poor BP control 30 days and 1 year after haemorrhagic stroke. ${ }^{11}$ In this study, BP was at goal (less than 140/90) for $47.2 \%$ of participants at 30 days and for $41.7 \% 1$ year after stroke. Current practice guidelines give clear recommendations for BP treatment after stroke; however, these studies suggest that the recommendations are not effectively implemented in clinical practice. ${ }^{12}$

\section{Interventions for BP control}

Multiple behavioural, psychosocial, environmental and physiological factors contribute to risk factor control in stroke survivors. In addition to race and socioeconomic status, medication adherence, self-efficacy, marital status and level of independence are associated with BP control. ${ }^{9} 10$ 13-15 Physiological factors such as duration of hypertension, differential response to medications according to race and ethnicity, and medical comorbidities such as sleep apnoea and chronic kidney disease may also be associated with more resistant hypertension. ${ }^{16-19}$ The complexity of these factors and their potential interactions may help explain why BP interventions have been largely ineffective in stroke survivors.

A Cochrane review of randomised clinical trials for poststroke risk factor management revealed that isolated behavioural and interventions did not impact BP control. ${ }^{20}$ Pooled analyses of organisational interventions such as those incorporating revisions of professional roles, collaboration of multidisciplinary teams, integrated care services, and/or knowledge and quality management protocols demonstrated trends towards improvements in $\mathrm{BP}$ control. The effect sizes in the trials assessing change in BP were small (less than $4 \mathrm{mmHg}$ change in systolic BP, SBP) and the trials had moderately small sample sizes. Effect sizes might be larger if multiple aspects of care delivery and patient education are addressed in a single intervention. Our aim is to assess the effectiveness of an organisational intervention on BP control in a new type of clinic designed for patients with stroke.

\section{Stroke Transitions Education and Prevention clinic}

The Stroke Transitions Education and Prevention (STEP) clinic was developed with the goal of providing integrated care for secondary stroke prevention and stroke complication assessment and management. The patients are managed by a stroke prevention neurologist and a stroke nurse practitioner with training in family medicine. The care team provides stroke education to patients and caregivers, manages uncontrolled risk factors according to protocols and evidence-based guidelines, and supports transitions back into the community.

Patients are referred from a Joint Commission-certified Comprehensive Stroke Center (CSC) at Memorial Hermann Hospital adjacent to the McGovern Medical School in the University of Texas Health Sciences Center (UTHealth). The programme serves a diverse population that is approximately $50 \%$ non-Hispanic white, $30 \%$ African-American and 15\% Hispanic American. At the STEPs Georgetown clinic, patients are referred the adjacent MedStar Georgetown University Hospital in Washington DC, which is also a CSC, and serves a population, that is, $55 \%$ African-American, $42 \%$ white and 3\% Asian. The STEP programme is not the standard of care for patients with stroke, but patients are assigned to the STEP programme based on provider availability. Patients with stroke who are not referred to the STEP programme are scheduled with another neurologist in the outpatient neurology clinic or with a community neurologist. The STEP programme has potential to impact risk factor reduction for secondary stroke prevention.

\section{Study objectives}

The primary objective is to compare the effectiveness of poststroke management in the STEP clinic versus usual care on BP reduction among patients with uncontrolled BP. We hypothesise that the STEP clinic will be more effective than usual care at decreasing mean daytime ambulatory SBP by 6 months after randomisation.

Secondary objectives will assess the impact of STEP care on additional BP and stroke outcomes. These outcomes include the proportion of patients achieving $\mathrm{BP}$ control, the proportion of participants monitoring BP, BP medication adherence, BP self-efficacy and body mass index (BMI). We will also assess the occurrence of cardiovascular events (composite stroke recurrence, myocardial infarction and vascular death) and use 
Bayesian analysis to assess the probability of a difference in this outcome between STEP clinic and usual care. We plan to assess modifying effects of race/ethnicity on the relationship between the study intervention and BP outcomes including mean ambulatory BP and the proportion achieving BP control at 6 months. Finally, we will compare the health system costs of follow-up care in the STEP clinic to the costs of usual care. Our goal is to estimate the incremental costs of care with STEP per additional patient with controlled $\mathrm{BP}$ according to the American Heart Association guidelines.

\section{METHODS AND ANALYSIS}

The STEP for blood pressure reduction study is a randomised comparative effectiveness trial with a parallel arm design. Patients are recruited from the Memorial Hermann Hospital System in Houston, Texas and from the MedStar Georgetown University Hospital (site initiated in September 2017).

The trial will be completed in December 2018. We used the Standard Protocol Items: Recommendations for Interventional Trials reporting guidelines for this protocol manuscript. ${ }^{21}$

\section{Eligibility criteria}

Inclusion criteria are as follows: age $\geq 18$, hospitalisation for clinical ischaemic stroke, hypertensive haemorrhage or transient ischaemic attack (TIA), hypertension as evidenced by (1) history of hypertension, (2) hospital $\mathrm{BP} \geq 140 / 90 \mathrm{~mm} \mathrm{Hg}$ on two or more occasions during hospitalisation or (3) discharge home on BP medication; willingness and ability to follow up in the stroke clinic, discharge home or to short stay inpatient rehabilitation $(<2$ weeks) after stroke and uncontrolled clinic BP 2 weeks after hospital discharge. A transient ischaemic attack diagnosis requires agreement two neurologists. Patients are excluded if they meet any of the following criteria: modified Rankin Scale (mRS) >3 at time of enrolment, terminal illness, chronic kidney disease stage 4 or greater [estimated glomerular filtration rate $($ eGFR) $<30$; or end stage renal diease (ESRD) ], pregnancy, symptomatic flow limiting carotid stenosis without plan for intervention prior to initial clinic visit, rare stroke aetiology presumed unrelated to atherosclerotic risk factors (vasculitis, malignancy associated, substance abuse). Patients who were enrolled in other interventional studies were no eligible for the trial.

\section{Consent and randomisation}

Study procedures are depicted in figure 1. Sequential eligible patients are approached for study participation prior to hospital discharge or are called on the telephone shortly after discharge. Informed consent is obtained by research coordinators or study coinvestigators prior to discharge for patients approached in the hospital and in the outpatient clinic for patients contacted via telephone. If a patient is unable to give consent due to cognitive impairment, consent is obtained from a legally authorised representative. The final eligibility criterion (uncontrolled BP) is assessed at the initial clinic visit which occurs between 1 week and 30 days of hospital discharge. Uncontrolled BP was initially defined as sitting automated office BP of $\geq 135 / 85 \mathrm{~mm} \mathrm{Hg}$ which is equivalent to $\geq 140 / 90$ by standard office BP assessment. ${ }^{22} 23$ Following release of the 2017 Hypertension Guidelines, uncontrolled $\mathrm{BP}$ was redefined as $\mathrm{BP} \geq 130 / 80$ by standard office BP, so this eligibility criterion was changed to $\mathrm{BP} \geq 125 / 75$ by automated office $\mathrm{BP}^{24}$ This change was implemented in January 2018 (protocol V.3-updated on ClinicalTrials.gov).

On presentation for the initial clinic screening visit, outpatient stroke clinic medical assistants (MAs) perform the initial vital signs assessment. Attended BP measures are obtained by MAs with a calibrated automated BP machine Welch Allen Spots Vital Signs (4200-88E). The MAs also obtain weight and height, then bring the patients to the dedicated research suite for further evaluation with the research coordinator. After the patient and/or caregiver complete the demographic questionnaire, the caregiver(s) are asked to leave the room for the automated $\mathrm{BP}$ assessment using BpTRU, one of the most extensively studied automated BP machines. ${ }^{25-27}$ The research coordinator applies an appropriately sized cuff to the patient's left upper arm. The patient is positioned so that his or her feet are flat on the floor, back is supported and legs are uncrossed. The left arm is placed on a table at chest level in the supine position. The research staff observes the first BP recording to assess adequacy and leaves the patient alone in the room for the remaining five measurements. The machine is programmed to take six measurements 2 min apart and to discard the first. The research staff returns after $10 \mathrm{~min}$ to record the BpTRU readings on clinic screening forms. The average of the last five readings is used to determine final eligibility. Patients who are found to have markedly elevated sitting BP at the baseline visit $(\geq 170 / 105)$ have an immediate visit with a medical doctor (MD) or stroke nurse practitioner (NP) before randomisation.

Following completion of baseline forms (table 1), eligible patients are randomised to STEP clinic or usual care using the REDCap randomisation module. A statistician who is not involved in patient allocation (Pedroza) developed the random sequence with 1:1 allocation ratio and block sizes of 4-8 and loaded the sequence into REDCap. The allocation sequence is not accessible to any other study investigators. Stratification variables include study site, SBP at the time of randomisation (SBP $<155 \mathrm{vs}$ $\geq 155$ ) and insurance status. The principal investigators and research coordinators are not blinded to group assignment. The coinvestigator reading the ambulatory blood pressure monitoring for the final outcome assessment is blinded to group assignment. The statistician is blinded to group assignment.

Following randomisation, participants are scheduled to follow up in the STEP clinic or usual care within 2 weeks 


\section{HOSPITAL}

Prescreen

Eligible patients consented and scheduled for 2 week visit at Stroke Outpatient Clinic.

\section{OUTPATIENT CLINIC}

\begin{tabular}{|c|}
\hline Screen \\
Vital signs assessed including \\
weight, height, and standard BP. \\
Research coordinator collects \\
demographic form and measures \\
automated office BP using \\
BpTRU (unattended automated BP \\
machine). If BpTRU $\geq 135 / 85$ \\
eligible for randomization*. If \\
BpTRU $\geq 170 / 105$, immediate MD \\
or NP visit.
\end{tabular}

RANDOMIZATION

\section{STEP CLINIC}

Individualized stroke plan developed Timely BP log review based on BP range Omron BP cuff provided for BP monitoring

Medication non-adherence screen

Sleep apnea screen

Diet counseling

\section{MONTH FOLLOW UP CALL}

3 Months

Assess adverse events, major

cardiovascular events, and healthcare utilization.

\section{USUAL CARE}

Stroke clinic visit

Risk factor and complication assessment, education, and management done according to provider.

\section{MONTH FOLLOW UP VISIT}

\begin{tabular}{|c|}
\hline 6 Months \\
Vital signs assessed including weight \\
height, standard BP, and BpTRU. \\
Primary and secondary outcomes \\
assessed. Ambulatory BP monitor \\
provided. Claims data for cost analysi \\
collected. \\
\hline
\end{tabular}

\section{MONTH INTER VAL CALLS}

\begin{tabular}{c} 
Safety/CV Outcomes \\
Assess adverse events, major \\
cardiovascular events, and healthcare \\
utilization every three months until \\
data lock. \\
\hline
\end{tabular}

\section{DATA LOCK}

*Inclusion criteria changed to BPTRU $\geq 125 / 75$ January 2018 .

Figure 1 This figure illustrates the timeline and procedures for study screening, enrolment and follow-up. BP, blood pressure; CV, cardiovascular; STEP, Stroke Transitions Education and Prevention. 
Table 1 Data and outcome assessment schedule

\begin{tabular}{|c|c|c|c|c|c|c|c|}
\hline & Prescreening & Screening & Randomisation & Stroke clinic & $3 \mathrm{mos}$ & $6 \mathrm{mos}$ & $\begin{array}{l}\text { Safety/ } \\
\text { cardiovascular outcomes }\end{array}$ \\
\hline Visit no & Vo & V1 & V1 & V2 & V3 & V4 & V5-V12 \\
\hline Timeline & -30 to -7 days & 0 & 0 & 2 wks & & $6 \mathrm{mo}$ & Every 3 mos until data lock \\
\hline Visit window & & & & \pm 2 wks & \pm 2 wks & \pm 2 wks & \pm 2 wks \\
\hline Location & Hospital & Clinic & Clinic & Clinic & Clinic/phone & Clinic & Phone \\
\hline \multicolumn{8}{|l|}{ Procedures and forms } \\
\hline $\begin{array}{l}\text { Prescreen (hospital) } \\
\text { eligibility/consent }\end{array}$ & $x$ & & & & & & \\
\hline $\begin{array}{l}\text { Screen (clinic) } \\
\text { Demographic } \\
2 \text { wks clinic BP } \\
\text { BMI }\end{array}$ & & $\begin{array}{l}X \\
X \\
X\end{array}$ & & & & & \\
\hline
\end{tabular}

Randomisation visit (prior

to randomisation)

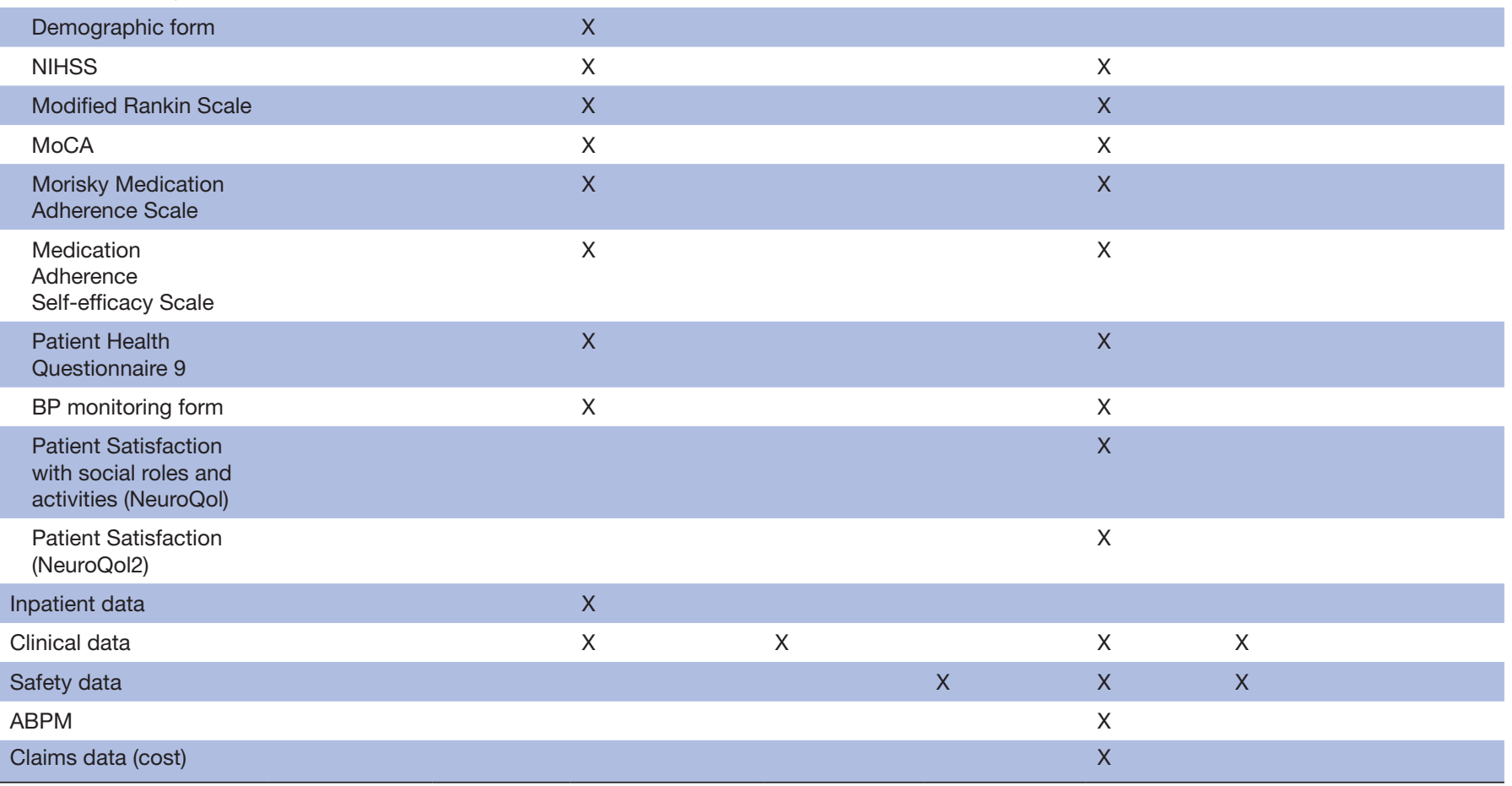

ABPM, ambulatory blood pressure monitoring; BMI, body mass index; BP, blood pressure; mo, month; MoCA, Montreal Cognitive Assessment; NIHSS, National Institutes of Health Stroke Scale; wk, week.

of randomisation. Patients randomised to the STEP clinic receive a $\mathrm{BP}$ monitor, recommendations for self-monitoring, a folder contained information about stroke risk factors, a BP monitoring brochure, a BP log, a Mediterranean diet brochure and pyramid, and instructions for follow-up. Patients randomised to usual care receive the educational folder and are encouraged to monitor BP.

\section{Study arms}

The STEP arm includes patients randomised to attend the STEP clinic for poststroke risk factor management. At the initial STEP clinic visit, hospital records are reviewed, and an individualised stroke care plan is developed with the patient (and caretakers if present) based on best-practice guidelines. The BP $\log$ is reviewed, and adjustments to medications are made based on BP goals.
All patients are screened for medication non-adherence and counselled on the importance of adherence and BP monitoring. The most affordable medications are used as indicated. The BP regimen is reviewed to decrease polypharmacy and multiple daily dosing of medications. All patients are screened for sleep apnoea given its association with uncontrolled BP and stroke risk. Patients are counselled and given information on the Mediterranean diet and the importance of decreased sodium intake and exercise for stroke prevention. If BP is not at goal, medications are adjusted and a BP check or telephone follow-up of 2-4 weeks is scheduled according to BP range (4 weeks for home SBP 125-154; 2 weeks for SBP 155-174; telephone follow-up and 2-week clinic follow-up for $\mathrm{SBP} \geq 175$ ). If $\mathrm{BP}$ is at goal at the initial visit, patients 
will be scheduled for follow-up in 3 months, but BP records are reviewed monthly. More urgent follow-up may also be scheduled according to other factors including depression, clearance for return to work after neuropsychological testing or sleep study follow-up. The care plan is shared with primary providers and patients are referred to a primary provider if they do not yet have one.

Participants randomised to usual care are scheduled to attend an initial stroke fellow or stroke attending clinic. Risk factor and complication assessment, education, and management are done according to provider practices. Recommendations are sent to referring/primary providers and follow-up is according to provider practices.

\section{Outcome measures and assessment points}

The primary outcome is the difference in mean daytime ambulatory SBP at 6 months between groups. Secondary outcomes include the difference in mean daytime ambulatory diastolic blood pressure (DBP) at 6 months; mean ambulatory night-time SBP and DBP at 6 months; proportion of patients achieving $\mathrm{BP}$ control at 6 months using ambulatory and sitting BPs; BP medication adherence at 6 months using Morisky Medication Adherence Scale ${ }^{28}$; depressive symptoms as assessed by Patient Health Questionnaire 9 at 6 months $^{29}$; per cent of patients monitoring $\mathrm{BP}$ at 6 months; satisfaction with social roles and activities, as measured by NeuroQOL short form at 6 months ${ }^{30}$; differences in patient satisfaction with stroke clinic at 6 months using Consumer Assessment of Healthcare Providers and Systems (CAPHS) surveys ${ }^{31}$; differences in self-efficacy at 6 months ${ }^{32}{ }^{33}$; differences in composite cardiovascular events from enrolment to and study lock; differences in harmful events during the intervention period; direct medical costs and cost-effectiveness.

Baseline demographic and clinical characteristics are abstracted from inpatient charts, supplemented by a demographic case report form (CRF) collected during the initial visit. Demographic variables include age, sex, self-reported race, self-reported ethnicity, level of education, household income, insurance status and marital status. Clinical variables include stroke subtype, stroke aetiology, prior stroke or TIA, treatment with intravenous tissue plasminogen activase (tPA), treatment with intra-arterial intervention, admission National Institutes of Health Stroke Scale, prestroke (mRS), presence of stroke risk factors (hypertension, diabetes mellitus, tobacco use, hyperlipidaemia, obstructive sleep apnoea, atrial fibrillation, coronary artery disease, systolic heart failure, substance abuse) other medical comorbidities, BMI and number of prescribed medications on admission and at discharge.

Total hospital and clinic costs will be assessed from a healthcare system perspective. Hospital costs will be estimated by multiplying charges obtained from the 15 Memorial Hermann Health System hospitals and from Georgetown University Hospital by their department-specific cost-to-charge ratios specified in their annual Medicare cost report. Clinic costs will be estimated by applying the relative value unit (RVU) based method to UTH and Georgetown University billing data. Every 3 months until study end, patients will be called to identify any outside hospital and clinic services. In addition, the primary care medical records for those followed outside our centre will also be sought. The costs for care received outside will be estimated based on the cost for these services at our centre. Medication costs will be estimated based on the prescriptions for each patient and the average wholesale prices in the Red Book Drug References. The STEP programme costs will also include the estimated cost for personnel time spent providing the programme (above that for usual care) based on time-and-motion studies and activity and phone call logs. Time costs will be estimated based on staff salary and fringe data. Additional costs associated with the intervention, for example, costs of print materials, will also be estimated and added to the medical and personnel costs to obtain the total cost of the intervention.

Provider recommendations will be ascertained from the clinic electronic records. In addition to scheduled clinic visits, participants will attend follow-up research assessments 6 months after randomisation. At the 6 -month visit, the MAs perform the vitals assessment including weight, height and attended BP. A research coordinator measures sitting BP using BpTRU machine. Patients complete outcome assessments (table 1). Participants are also sent home with an ambulatory BP monitor and a prepaid FedEx box for return of the monitor. The monitor is mailed back to the research coordinator, and data are downloaded and analysed by an investigator blinded to patient group. Mean daytime ambulatory SBP is assessed as the mean in SBP measurements taken from 8:00 hours to 20:00 hours.

We will assess major clinical outcomes including recurrent stroke, myocardial infarction and vascular death every 3 months via telephone call (or follow-up visit) from enrolment until data lock. Hospital and emergency department (ED) records will be requested if reported at 3-month patient encounters. Additional safety outcomes including syncope, falls or dizziness/hypotension requiring $\mathrm{ED}$ visit/hospitalisation will also be assessed every 3 months until 6 months. At the 6-month follow-up visits, patient clinic records are requested from primary providers to aid in cost analysis.

\section{Participant retention}

Participants are provided with parking passes for research and clinic visits and are provided with compensation for their time for research visits. Home visits for final outcome assessments are offered if participants cannot travel to the clinic. If participants cannot be located for follow-up, we attempt to reach listed emergency contacts before mailing a letter (prior permission) to their homes. We also send holiday cards and newsletters to participants to promote retention.

\section{Statistical analysis and sample size calculations}

Intention-to-treat analysis will be performed using STATA software V.14. ${ }^{34}$ For the primary analysis, linear regression 
will be used to compare the difference in mean daytime ambulatory SBP between groups using ambulatory SBP as the dependent variable and treatment group, baseline SBP (sitting/continuous) and insurance status as independent variables. As a secondary analysis of the primary outcome, we will assess treatment effect modification by race/ethnicity using the same linear regression model and introducing an interaction term. Secondary analyses will be used to evaluate additional clinical, behavioural and safety outcomes.

Linear regression models will be used for continuous variables and logistic regression models will be used for dichotomous variables. For behavioural outcomes (medication adherence/self-efficacy), Wilcoxon rank sum or ordinal regression will be used if proportional hazards assumptions are met. Costs will be compared using multilevel generalised estimating equations models with gamma distribution and log link. For safety outcomes, we will use a Poisson regression model; and for composite vascular events, we will also use Bayesian analysis to estimate probability of an event. All models will be adjusted for stratifying variables.

The cost-effectiveness of the programme will be estimated by dividing the incremental costs of the STEP programme relative to usual care by the incremental number of patients with controlled BP at 6 months. We will also perform sensitivity analyses and probabilistic sensitivity of plausible ranges for costs and effectiveness.

In order to detect a $5 \mathrm{~mm} \mathrm{Hg}$ difference in the change in mean ambulatory SBP from baseline to 6 months (power $0.8, \alpha 0.05)$ using an $11.5 \mathrm{~mm} \mathrm{Hg}$ SD for SBP change, we would need to retain 84 patients in each group. The $5 \mathrm{~mm}$ $\mathrm{Hg}$ difference was chosen because a meta-analysis of $\mathrm{BP}$ reduction trials revealed OR for recurrent stroke of 0.78 $(0.68,0.9)$ with mean change SBP of $5.1 \mathrm{~mm} \mathrm{Hg.}{ }^{7}$ Assuming attrition of $15 \%$, we will enrol 100 patients per group.

\section{Patient and public involvement}

The STEP clinic is designed as a patient-centred care model, which is informed by informal assessments of patient and caregiver preferences and goals during clinic visits. Patients were not formally involved involved in the trial design or conduct. While the primary outcome is a measure of BP, secondary outcomes include measures that relate to quality of life and patient satisfaction. Furthermore, the CAPHS surveys are used to assess patient satisfaction with care provided in the STEP clinic relative to usual care. This survey will assist in our assessment of the burden of the intervention to patients. On study completion and analysis of outcomes, a newsletter will be sent to participants to inform them of study results.

\section{ETHICS AND DISSEMINATION}

The study design, risks and benefits, and patient confidentiality were judged rigorously. The use of protected health information (PHI) is minimised and any electronic files containing PHI are stored in password-protected documents on secure servers. Paper CRFs and consents are stored in locked cabinets in a locked office. The files containing PHI will be retained for 5 years after trial completion. The final dataset will be available to the study principal investigator, the study statistician and coinvestigators by request. A manuscript with the results of the study will be published in a peer-reviewed journal. Trial results will be communicated to participants via a newsletter.

Patients are eligible regardless of insurance status or financial ability to follow up in the clinic and we guarantee all patients, regardless of randomisation assignment, one free clinic visit with a neurologist. If patients report stroke signs or symptoms, have dangerously elevated BP or report other critical symptoms (chest pain, shortness of breath) during the course of the study, they are treated or referred as appropriate, regardless of clinic assignment.

\section{Data monitoring and management}

Baseline forms and outcome assessments are obtained using paper CRFs and are subsequently entered a secure REDCap database. The database structure includes range checks for data values, and each data field contains a specific description of the data element including where to find the data in the medical record. Accurate entry of data from paper CRFs into REDCap is verified by coinvestigators. Principal investigators review data fields abstracted by research assistants and coordinators.

\section{DISCUSSION}

Despite the increasing prevalence of stroke in the coming years, there is little emphasis or research on organising systems of care for stroke survivors. Multiple behavioural, psychosocial, environmental and physiological factors contribute to risk factor control. ${ }^{13}{ }^{35-38}$ Hypertension is a major risk factor for recurrent stroke, and BP reduction is associated with decreased risk of stroke recurrence. ${ }^{1}$ However, many stroke survivors remain with poorly controlled BP after their initial stroke. ${ }^{910}$ The complexity of these risk factors and their potential interactions are not well understood and could explain why isolated BP interventions have been largely ineffective in patients with stroke. Poststroke care should address the unique needs of stroke survivors and prioritise risk factor management for prevention of recurrent stroke. The main goal of the STEP clinic is to implement an organisational intervention on BP control which integrates the various known stroke risk factors into a new type of clinic designed for patients with stroke.

If the STEP clinic care is found to be more effective in reducing $\mathrm{BP}$, it may provide a means to improve poststroke care. A cost analysis comparing cost of the STEP clinic to that of usual care would determine the feasibility of introducing this unique approach to integrated poststroke care as a standard. The STEP clinic could provide improvements in poststroke care, risk factor management and stroke recurrence prevention. This research is needed to determine whether the STEP clinic is more 
effective in managing stroke risk factors and improving stroke outcomes in comparison to usual care.

\section{Author affiliations}

${ }^{1}$ Department of Neurology, McGovern Medical School at the University of Texas Health Science Center at Houston, Houston, Texas, USA

${ }^{2}$ University of Texas Health Sciences Center, Institute for Stroke and Cerebrovascular Disease, Houston, Texas, USA

${ }^{3}$ Department of Pediatrics, McGovern Medical School at the University of Texas Health Science Center at Houston, Houston, Texas, USA

${ }^{4}$ Department of Neurology, Georgetown University Medical Center, Washington, Washington, DC, USA

Acknowledgements We acknowledge Kim Vu, Shawanda Miller and Norma Hunter for their assistance with caring for patients at the Memorial Hermann Hospital and in the McGovern Medical School Neurology clinic. We acknowledge Wesley Horton, Amanda Lantzy and Ashley Carlson-Chalifoux for assistance with recruiting and caring for patients at the Georgetown University Medical Center site.

Contributors AS is the principal investigator for the study. She is responsible for the study design and for overseeing data acquisition. She participated in drafting the manuscript. EH is a research assistant who participates in data acquisition and abstraction. She participated in manuscript drafting. GC is a research coordinator who participates in patient recruitment, enrolment, follow-up and data acquisition, and who revised the manuscript for important intellectutal content. M0 is a nurse practitioner who participates in patient recruitment, enrolment and follow-up. She participated in study design and manuscript drafting. EBA participates in study design regarding cost-effectiveness. She revised the manuscript for important intellectual content. CP is a statistician who participated in the design of the study and drafting of the manuscript. MCD is the principal investigator at the Georgetown University Medical Center. She participated in study design and participates in data acquisition. She revised the manuscript for important intellectutal content. JS participated in study design and participates in data analysis and acquisition. He revised the manuscript for important intelectual content. JET participated in study design. He contributed important intellectual content to the manuscript. SIS participated in study design and contributed important intellectual content to the manuscript.

Funding This research was funded by a KL2 award received from the University of Texas Health Science Center at Houston's Center for Clinical and Translational Sciences (HSC-MS15-046).

Disclaimer The funder did not play a role in the study design, data collection, data analysis, interpretation of data, writing of the report or the decision to submit the paper for publication.

Competing interests None declared.

Patient consent Not required.

Ethics approval Ethical approval was obtained by the Institutional Review Boards at the McGovern Medical School in Houston (10/2015) and by the Georgetown University School of Medicine (11/2017).

Provenance and peer review Not commissioned; externally peer reviewed.

Open access This is an open access article distributed in accordance with the Creative Commons Attribution Non Commercial (CC BY-NC 4.0) license, which permits others to distribute, remix, adapt, build upon this work non-commercially, and license their derivative works on different terms, provided the original work is properly cited, appropriate credit is given, any changes made indicated, and the use is non-commercial. See: http://creativecommons.org/licenses/by-nc/4.0/.

\section{REFERENCES}

1. Benjamin EJ, Blaha MJ, Chiuve SE, et al. Heart disease and stroke statistics-2017 Update: a report from the American heart association. Circulation 2017;135:e146-e603.

2. Mozaffarian D, Benjamin EJ, Go AS, et al. Heart disease and stroke statistics-2015 update: a report from the American Heart Association. Circulation 2015;131:e29-e322.

3. Ovbiagele B, Goldstein LB, Higashida RT, et al. Forecasting the future of stroke in the united states: a policy statement from the American Heart Association and American Stroke Association. Stroke 2013;44:2361-75.
4. Petty GW, Brown RD, Whisnant JP, et al. Ischemic stroke subtypes : a population-based study of functional outcome, survival, and recurrence. Stroke 2000;31:1062-8.

5. Hackam DG, Spence JD. Combining multiple approaches for the secondary prevention of vascular events after stroke: a quantitative modeling study. Stroke 2007;38:1881-5.

6. Group PC. Post-stroke antihypertensive treatment study. A preliminary result. Chin Med J 1995;108:710-7.

7. Liu L, Wang Z, Gong L, et al. Blood pressure reduction for the secondary prevention of stroke: a Chinese trial and a systematic review of the literature. Hypertens Res 2009;32:1032-40.

8. PROGRESS Collaborative Group. Randomised trial of a perindoprilbased blood-pressure-lowering regimen among 6,105 individuals with previous stroke or transient ischaemic attack. Lancet 2001;358:1033-41.

9. Brenner DA, Zweifler RM, Gomez CR, et al. Awareness, treatment, and control of vascular risk factors among stroke survivors. J Stroke Cerebrovasc Dis 2010;19:311-20.

10. White CL, Pergola PE, Szychowski JM, et al. Blood pressure after recent stroke: baseline findings from the secondary prevention of small subcortical strokes trial. Am J Hypertens 2013;26:1114-22.

11. Zahuranec DB, Wing JJ, Edwards DF, et al. Poor long-term blood pressure control after intracerebral hemorrhage. Stroke 2012;43:2580-5.

12. Kernan WN, Ovbiagele B, Black HR, et al. Guidelines for the prevention of stroke in patients with stroke and transient ischemic attack: a guideline for healthcare professionals from the American Heart Association/American Stroke Association. Stroke 2014;45:2160-236.

13. Ireland SE, Arthur HM, Gunn EA, et al. Stroke prevention care delivery: predictors of risk factor management outcomes. Int J Nurs Stud 2011;48:156-64.

14. Dave GJ, Bibeau DL, Schulz MR, et al. Predictors of uncontrolled hypertension in the Stroke Belt. J Clin Hypertens 2013;15:562-9.

15. Aronow WS, Fleg JL, Pepine CJ, et al. ACCF/AHA 2011 expert consensus document on hypertension in the elderly: a report of the American college of cardiology foundation task force on clinical expert consensus documents developed in collaboration with the American Academy of Neurology, American Geriatrics Society, American Society for Preventive Cardiology, American Society of Hypertension, American Society of Nephrology, Association of Black Cardiologists, and European Society of Hypertension. J Am Soc Hypertens 2011;5:259-352.

16. Park IU, Taylor AL. Race and ethnicity in trials of antihypertensive therapy to prevent cardiovascular outcomes: a systematic review. Ann Fam Med 2007;5:444-52.

17. Wright JT, Dunn JK, Cutler JA, et al. Outcomes in hypertensive black and nonblack patients treated with chlorthalidone, amlodipine, and lisinopril. JAMA 2005;293:1595-608.

18. Bradley TD, Floras JS. Obstructive sleep apnoea and its cardiovascular consequences. Lancet 2009;373:82-93.

19. Diminic-Lisica I, Popovic B, Rebic J, et al. Outcome of treatment with antidepressants in patients with hypertension and undetected depression. Int J Psychiatry Med 2014;47:115-29.

20. Lager KE, Mistri AK, Khunti K, et al. Interventions for improving modifiable risk factor control in the secondary prevention of stroke. Cochrane Database Syst Rev 2014;5:CD009103.

21. Chan AW, Tetzlaff JM, Altman DG, et al. SPIRIT 2013 statement: defining standard protocol items for clinical trials. Ann Intern Med 2013;158:200-7.

22. Mancia G, Fagard R, Narkiewicz K, et al. 2013 ESH/ESC Guidelines for the management of arterial hypertension: the Task Force for the management of arterial hypertension of the European Society of Hypertension (ESH) and of the European Society of Cardiology (ESC). J Hypertens 2013;31:1281-357.

23. Myers MG, Kaczorowski J, Paterson JM, et al. Thresholds for diagnosing hypertension based on automated office blood pressure measurements and cardiovascular risk. Hypertension 2015;66:489-95.

24. Whelton PK, Carey RM, Aronow WS, et al. ACC/AHA/AAPA/ABC/ ACPM/AGS/APhA/ASH/ASPC/NMA/PCNA Guideline for the Prevention, Detection, Evaluation, and Management of High Blood Pressure in Adults: A Report of the American College of Cardiology/ American Heart Association Task Force on Clinical Practice Guidelines. Hypertension 2017.

25. Beckett L, Godwin M. The BpTRU automatic blood pressure monitor compared to 24 hour ambulatory blood pressure monitoring in the assessment of blood pressure in patients with hypertension. BMC Cardiovasc Disord 2005;5:18.

26. Myers MG, Godwin M. Automated office blood pressure. Can $J$ Cardiol 2012;28:341-6. 
27. Myers MG, Valdivieso M, Kiss A. Use of automated office blood pressure measurement to reduce the white coat response. $J$ Hypertens 2009;27:280-6.

28. Morisky DE, Ang A, Krousel-Wood M, et al. Predictive validity of a medication adherence measure in an outpatient setting. $J$ Clin Hypertens 2008;10:348-54.

29. Williams LS, Brizendine EJ, Plue L, et al. Performance of the PHQ-9 as a screening tool for depression after stroke. Stroke 2005;36:635-8.

30. Gershon RC, Lai JS, Bode R, et al. Neuro-QOL: quality of life item banks for adults with neurological disorders: item development and calibrations based upon clinical and general population testing. Qual Life Res 2012:21:475-86.

31. Weinick RM, Quigley DD, Mayer LA, et al. Use of CAHPS patient experience surveys to assess the impact of health care innovations. Jt Comm J Qual Patient Saf 2014;40:418-27.

32. Lorig KR SA, Ritter P, Gonzalez V, et al; Outcome measures for health education and other health care interventions. Thousand Oaks, CA SAGE Publications, 1996.
33. Warren-Findlow J, Seymour RB, Brunner Huber LR. The association between self-efficacy and hypertension self-care activities among African American adults. $J$ Community Health 2012;37:15-24.

34. Boston RC, Sumner AE. STATA: a statistical analysis system for examining biomedical data. Adv Exp Med Biol 2003;537:353-69.

35. World Health Organization. Adherence to long-term therapies: evidence for action, 2003.

36. Schroeder K, Fahey T, Ebrahim S. Interventions for improving adherence to treatment in patients with high blood pressure in ambulatory settings. Cochrane Database Syst Rev 2004;2:CD004804.

37. Briesacher BA, Gurwitz JH, Soumerai SB. Patients at-risk for costrelated medication nonadherence: a review of the literature. $J$ Gen Intern Med 2007;22:864-71.

38. Boutin-Foster C, Offidani E, Kanna B, et al. Results from the Trial Using Motivational Interviewing, Positive Affect, and Self-Affirmation in African Americans with Hypertension (TRIUMPH). Ethn Dis 2016;26:51-60 